\title{
Philosophiques
}

\section{Psychanalyse et féminité}

\section{Le désir de l'un - inscrit sur l'autre-femme - pour l'Un.}

\section{Marie-Christine Brousson-Rosay}

Volume 12, numéro 1, printemps 1985

URI : https://id.erudit.org/iderudit/203278ar

DOI : https://doi.org/10.7202/203278ar

Aller au sommaire du numéro

Éditeur(s)

Société de philosophie du Québec

ISSN

0316-2923 (imprimé)

1492-1391 (numérique)

Découvrir la revue

\section{Citer cet article}

Brousson-Rosay, M.-C. (1985). Psychanalyse et féminité : le désir de l'un inscrit sur l'autre-femme - pour l'Un. Philosophiques, 12(1), 177-190.

https://doi.org/10.7202/203278ar

\section{Résumé de l'article}

La femme tableau et/ou miroir de l'homme - telle qu'il la crée, l'aime renvoie de celui-ci l'image inversée : forme de l'« autre » elle montre dans ce qui se dit d'elle le désir de l'« un » (homme) comme désir d'être l'un et l'autre (sexe), désir d'être le « Tout » ou, désir de l'« Un ». Plusieurs textes freudiens marquent sans ambiguité la " sexuation » du désir, marquent le désir comme principe mâle. Il s'agit ici de montrer, avec ces textes, les places et les articulations de l'un et l'autre sexe dans la dynamique du désir.
Ce document est protégé par la loi sur le droit d'auteur. L'utilisation des services d'Érudit (y compris la reproduction) est assujettie à sa politique d'utilisation que vous pouvez consulter en ligne.

https://apropos.erudit.org/fr/usagers/politique-dutilisation/ 


\title{
PSYCHANALYSE ET FÉMINITÉ \\ Le désir de l'un - inscrit sur l'autre-femme - pour l'Un. \\ par Marie-Christine Brousson-Rosay
}

\begin{abstract}
RÉSUMÉ. La femme tableau et/ou miroir de l'homme - telle qu'il la crée, l'aime - renvoie de celui-ci l'image inversée : forme de l' " autre " elle montre dans ce qui se dit d'elle le désir de l'" un " (homme) comme désir d'être l'un et l'autre (sexe), désir d'être le «Tout» ou, désir de l' « Un». Plusieurs textes freudiens marquent sans ambiguïté la « sexuation »du désir, marquent le désir comme principe mâle. Il s'agit ici de montrer, avec ces textes, les places et les articulations de l'un et l'autre sexe dans la dynamique du désir.
\end{abstract}

ABSTRACT. Woman as a picture and/or mirror of man - as he creates and loves her - gives an reversed image of him : she, a form of the "other", reveals in what is said of her the desire of "one" (man) as the desire to be both the one and the other (sex), the desire for "Whole"-ness, for "One"-ness. Several Freudian texts unambiguously mark the sexualisation of desire, desire as a male principle. What is shown here, with these texts, are the places and articulations of the one and the other sex in the dynamics of désire.

Le courage de ne garder aucune question sur le cœur, c'est cela qui fait le philosophe. Il doit être tel l'Oedipe de Sophocle qui, cherchant l'éclaircissement de son propre terrible destin, poursuit sans trêve son enquête alors même qu'il commence à entrevoir que des réponses résultera pour lui le plus horrible. Mais il se trouve que la plupart des hommes portent en eux une Jocaste qui, au nom de tous les dieux, supplie Oedipe de ne pas poursuivre sa recherche : et ils lui cèdent, et c'est 
pourquoi la philosophie en est encore au point où elle en est.
A. Schopenhauer (extrait d'une lettre à Goethe cité par C. Stein dans La mort d'Oedipe $)^{1}$.

Un jour, peut-être une nuit, l'autre-femme lut dans le discours amoureux de l'autre-homme :

“JE M'AIME QUAND TU DIS QUE TU M'AIMES "

Cet énoncé présente mon texte/lecture. Un sous-titre :

Le désir de l'un - inscrit sur l'autre-femme - pour l'Un.

Parvenu à la connaissance, on ne peut plus ni bien haïr ni bien aimer ; on demeure par delà la haine et l'amour. On s'est livré à l'investigation au lieu d'aimer (. . . ) on s'est livré à l'investigation au lieu d'agir, de créer. ${ }^{2}$

Ainsi, aimer, haïr c'est créer . . l'objet d'amour, non le connaître.

« Je » (la Loi) aime « moi » quand « tu » (l'autre-femme) dit que « $\mathrm{Tu}$ » (le Manque/écho de « je ») m’aime.

Que le même corps/même signifiant « tu » se lise à la fois sujet de l'énonciation et sujet de l'énoncé, fait que la Loi m'aime, me hait, fait que «tu " dis-paraît sous la forme du Manquel écho de « je ». Que tu dises que tu m'aimes et j'entends que c'est « $T u$ » qui m'aime par toi qui le dis. Tu es à la fois un autre corps-parlant et cet autre nécessaire à « je " marque signifiante de cette Loi (dite la Loi) qui fait de toi la bouche de mon désir, cette loi qui fait que — par toi qui le dis — je m'aime.

Quand tu dis que c'est toi qui m'aimes, me hais (me crées ?), ce n'est pas toi que j'entends m'aimer, me haïr, me créer objet d'amour mais la Loi. Pourtant, si tu ne dis ce que tu dis, la Loi ni ne me hait, ni ne m'aime, ni ne me crée.

Je ne t'aurai pas aimé moi qui, cette nuit (ou ce jour), aurai cherché à entendre ce que tu avais entendu de moi, moi qui aurai cherché à te connaître.

1. Denoël/Gonthier, 1977, p. 22.

2. Sigmund Freud, Souvenir d'enfance de Léonard de Vinci, Paris, Gallimard, 1927, p. 27. 
Le texte freudien met en scène deux créations, deux objets d'amour ? La Loi et le Manque plus un narrateur de l'Un et l'Autre, le "Moi ». Le Tout de la représentation est supporté par les planches (la scène au sens matériel du terme).

Dramatique à quatre termes dont :

- deux personnages, la Loi et le Manque,

- un narrateur, le Moi qui s'oublie dans les précédents,

- - un autre sur lequel se joue la pièce, les planches lieu d'inscription.

Si toute représentation produit, prescrit des effets d'identification, lesquels parmi nous, spectatrices, spectateurs, vont s'identifier aux planches?

Dans le texte freudien - encore - deux événements sont dits générateurs de théories/constructions, deux événements : la naissance et la mort créées objets d'amour et/ou de haine, deux pulsions.

\section{LA NAISSANCE-ÉVÉNEMENT, GÉNÉRATRICE DE LA THÉORIE/ CONSTRUCTION DE LA DIFFÉRENCE . . S SEXUELLE.}

Pour l'enfant, qui est dans ce premier cas le " sujet » théorisant, " le premier problème qui le préoccupe (. . .) n'est pas de savoir en quoi consiste la différence des sexes, mais la grande énigme : d'où viennent les enfants ? " ${ }^{3}$ C'est donc à partir d'une question sur l'origine — à être ou à maîtriser — que va s'élaborer la première théorie sexuelle, celle qui justement porte sur la (non) différence sexuelle, savoir : un même sexe - mâle - chez tous. "Les petits garçons ne mettent pas en doute que toutes les personnes qu'ils rencontrent ont un appareil génital semblable au leur ; ils ne leur est pas possible de concilier l'absence de cet organe avec l'idée qu'ils se font d'autrui (. . .) ils maintiennent même avec ténacité cette conviction, la défendent contre les faits contradictoires que l'observation ne tarde pas à leur révéler (. . .) $»^{4}$. Ayant appris que les enfants ont pour lieu d'origine le corps de la mère-femme, le jeune théoricien va « construire/théoriser » ce corps comme corps masculin, il va

3. Sigmund Freud, Trois essais sur la théorie de la sexualité, Paris, Gallimard, 1962, p. 91.

4. Ibid., p. 92. L'italique est de nous. 
faire d'elle un semblable mais porteur d'enfant, un " hommematernisé ».

Lorsque l'empiricité du corps de l'autre-femme se fera insistante, ne pouvant renoncer à sa place de référent, il le dira, ce corps, toujours semblable au sien mais cette fois en manque de pénis ; l'autre-femme sera construite " homme-en manque de pénis ", homme châtré, blessé, homme manqué. Cet autre corps sera lui toujours mais dans l'absence, le manque, un autre étrange, à la fois familier et inquiétant. De l'autre-femme, le jeune théoricien conservera deux images : "l'homme-maternisé »et "l'homme-sans pénis "; lui à la fois « l'un et l'autre " et lui en "manque-de l'un": son même.

Devant le sexe de la mère lu comme manque de pénis, le jeune théoricien va "vivre "sa division.

C'est du lieu de cette expérience que le texte freudien introduit la division du sujet ou plus justement l'avènement du sujet comme sujet-divisé, sujet-parlant/théorisant. Regardant le corps de l'autre-femme, le jeune théoricien construit la différence négative et son désir comme désir de "lui-même ": à la fois l'un et l'autre-corps sexué qu'il se représente : "homme-sous les traits d'une femme ", son objet d'amour. Il construit son désir comme nostalgie, première personne du futur antérieur : «j'aurai été ce tableau-miroir de moi-même qui est aussi mon ad-venir ». Mais, la beauté de "lui-même » s'effacera sous le Manque, signifiant de sa deuxième théorisation.

Le Manque sur l'autre-femme - son semblable - sera la marque/trace de ce qu'il aura été : le Tout - l'un dans l'apparence de l'autre - qui est aussi la forme-objet de son désir. Par (dans ?) l'autre-femme il cherchera la possibilité d'être Tout et ne rencontrera que le Manque. Il la voudra faire Tout(e)miroir de lui-même et, la regardant, il ne verra que le manque de lui, son négatif. Voulant retrouver l'Autre (ce qu'il aura été) il ne trouvera que le même, son manque, sa castration.

Modèle de son devenir originaire, "lui »-sous les traits d'une femme est dit le modèle de la beauté et . . . du Diable.

Deux textes freudiens mettent en scène cette double représentation de son désir - le désir ? - deux textes qui opposent l'une et l'autre des figures de son objet d'amour/haine. 
Dans Souvenir d'enfance de Léonard de Vinci (1910), le texte justifie l'art de Léonard par la fixation de ce dernier à sa représentation de la mère-toute c'est-à-dire ayant à la fois les attributs féminins, les seins, et l'attribut masculin, le pénis-en érection.

Le souvenir est le suivant : « ( . . .) étant encore au berceau, un vautour vint à moi, m'ouvrit la bouche avec sa queue et plusieurs fois me frappa avec cette queue entre les lèvres " 5 . Le pivot de l'interprétation est le vautour : « (. . .) divinité maternelle dotée par les Égyptiens d'un phallus ; son corps, que les seins caractérisent comme féminin, portait aussi un membre viril en état d'érection (. . .) $»^{6}$. Le vautour léonardesque n'est qu'un être " andro-gyne ", sa mère. Compte tenu des théories infantiles déjà décrites, le vautour du souvenir (du fantasme), est un homme au pénis en érection avec les attributs d'une femme, et, Léonard au berceau, un homme castré : l'autre-femme, recevant par la bouche ce qui va le faire être cet être-tout. Condensation des deux théories précédentes, le fantasme de Léonard est la représentation du désir d'être le «Tout-primitif» . . . à venir : Léonard se dit en manque . . . du Tout et désire, par contiguïté directe, le devenir. Les femmes qu'il va peindre ne seront que des exemplaires de ce qu'il veut être, à la fois l'un et l'autre. Le mystérieux sourire et l'étrange regard de Mona Lisa ne seront que les traces/marques de son appartenance au Tout, un pénis érigé caché sous le sourire/regard d'une «femme » : le Phallus.

«La Joconde, l'idéal féminin de Léonard enfin rencontré (. . .) Mona Lisa, représentation où Léonard s'est rencontré luimême ${ }^{7}$. Cette forme-objet de son désir se laissera deviner encore - dans la représentation de beaux " jeunes gens d'une délicatesse féminine, aux formes éfféminées ", l'une et l'autre des figures donnant à voir l'un et l'autre simultanément, donnant à voir le Tout de la puissance :

Ils (les beaux jeunes gens) ne baissent pas les yeux, mais nous regardent d'un regard mystérieusement vainqueur, comme $s$ 'ils connaissaient un grand triomphe de bonheur que l'on doit taire. (. . .) Une telle fusion bienheureuse de l'être mâle avec

5. Souvenir d'enfance de Léonard de Vinci, p. 49.

6. Ibid., p. 69 .

7. lbid., p. 102. 
l'être femelle, figure la réalisation des désirs de l'enfant autrefois fasciné par la mère. ${ }^{8}$

Être à la fois l'un et l'autre corps-séxué, tel est le désir originaire et à venir sans cesse du jeune théoricien. Sa production artistique (ou scientifique ?) sera la re-présentation de cet être — l'Être —, miroir de lui-même.

Mais, hélas, une telle fusion va aussi se dire le Diable, le monstre, l'« ab-ject ». Un texte freudien de 1923, Une névrose démoniaque au XVII $S .{ }^{9}$, offre au regard de tous les attributs du Diable : l'histoire est celle d'un peintre du nom de Christophe Haitzmann, lequel aurait contracté un double pacte avec le Diable. Il s'engage, dans un premier temps, à se considérer comme le fils du Diable pendant neuf ans et, dans un deuxième temps, à lui appartenir entièrement après sa mort.

Dans la rencontre de Haitzmann avec le Diable, ce dernier, après s'être présenté comme un honorable bourgeois, apparaît nu, difforme avec deux mamelles de femme. Or, le Diable, le texte nous le rappelle, est principe mâle, il est la représentation du père, mais pas n'importe quel père: Maître de l'enfer il est mâle, même plus que mâle, avec des cornes, une queue et un grand pénis-serpent; il est semblable au père de la " horde primitive ", le père ancestral tel qu'un autre texte freudien le présente (Totem et tabou, chapitre IV). Or, ce père, premier père, père-originaire, outre sa violence fondamentale, est maître de toutes les femmes : rapport de maîtrise par contiguïté directe, il est père/maître par elles. Ce père plus que mâle (maître et violent) est la représentation du mâle dans un rapport - corporel - avec des (une) femmes-mères et filles indifférenciées. Maître d'elles, le Père, père originaire, s'offre dans la forme-objet de l'un et l'autre (corps séxué) mais, par conjonction et non fusion. Le Diable, s'il fait signe vers le père ancestral par sa maîtrise de l'autre-sexe, ne donne à voir qu'un corps-fusion de deux. Andro-gyne monstrueux, il donne à voir simultanément " la femme " et " la mère " (l'andre sous les traits de la parthénos ?).

8. Ibid., p. 111 .

9. Sigmund Freud, Essais de psychanalyse appliquée, Paris, Gallimard, 1933. 


\section{Première figure : la femme.}

À partir de la deuxième théorie sexuelle qui fait du corps de l'autre-femme un corps castré/blessé, l'enfant mâle, de peur de perdre à son tour l'organe qui lui est si cher, va se soumettre au père-maître, c'est-à-dire adopter une " attitude féminine »: afin de ne pas être castré par le père, il se castre lui-même ; de cette position dite " féminine »va naître (sic) chez l'enfant mâle, un « fantasme de grossesse ».

Il n'y a peut-être pas, dans les constatations psychanalytiques sur la vie psychique de l'enfant, de partie qui semble, à un adulte normal, aussi déplaisante et aussi incroyable que l'attitude féminine du petit garçon envers le père et le fantasme de grossesse qui en découle.

(répugnance à la castration et/ou à l'attitude féminine ?)

Le rejet de l'attitude féminine est ainsi la conséquence de la lutte contre la castration, et il trouve (. . .) sa plus forte expression dans le fantasme contraire : châtrer le père lui-même, faire de lui une femme ${ }^{10}$.

Ainsi, le Diable, nous dit le texte, est la représentation du père châtré - le long pénis-serpent n'est pas l'équivalent de l'érection du vautour léonardesque mais la représentation de « la femme »: l'en plus représente l'en moins.

\section{Deuxième figure : la mère.}

Les seins développés (du Diable) sont la marque positive du sexe de la mère, à une époque où l'enfant ne connaît pas encore le caractère négatif de la femme, l'absence de pénis ${ }^{11}$.

D'un côté le monstre, l'abject : le sexe de la femme, de l'autre le sexe de la mère - deux dans un - se disant l'Un : les traits d'une femme sous lesquels se cache le pénis à l'impossible détumescence ; cela s'est déjà écrit/construit lors de la première théorisation : modèle du corps-sexe, perfection de l'Androgyne, cause de l'esthétique de Léonard . . . la beauté du Diable.

Dans le jeu des représentations, deux miroirs-sexes vont fasciner le sujet-de la théorie : le Tout-du sexe et le Manque,

10. Ibid., p. $233,234$.

11. Ibid. L'italique est de nous. 
les deux sous les traits d'une femme. Le pénis, quant à lui, de n'être signifié que par son manque, son absence ... de détumescence (toujours voilée), manque à être . . . vu, demeurera organe.

Dans le regard de l'autre-homme, reflet du miroir/tableau qu'elle est pour lui, l'autre-femme ne verra que le Tout-du sexe qu'il veut être : qu'il désire . . . avoir été, et/ou le manqueblessure de "lui-même ». Dans l'autre-homme, elle ne verra que le Tout-du sexe toujours déjà perdu, toujours déjà objet de désir et le Manque (blessure) représentation de ce désir, sa formeobjet. Elle le verra tel qu'il se dit en la disant mais ne se verra pas. Elle ne se verra pas mais agira le désir de « lui » en proposant ce que justement il ne veut pas : elle lui proposera " elle " les traits d'une femme - elle donnera ce qu'elle n'a pas - son corps-apparence - pour qu'enfin il puisse être (s'être) Tout.

Il le prendra ce corps-apparence en autant que le don d'elle à lui ne se signifiera pas ; il se l'appropriera dans le silence de son désir à elle, toute parole désirante - d'elle - ne pouvant que le renvoyer au manque (son manque) qu'il a construit . . . signe de son désir d'être le Tout-du sexe, soit le Tout-de la reproduction.

Ce dont le jeune théoricien veut faire l'économie est l'indispensable rencontre de l'autre-femme sexe pour sa re-production, production de lui-même.

Elle, toujours interpellée à être ce qui n'est pas là, effacée dans le silence de son désir (de rencontre/contact), femme absente, sans sens par le désir de " lui », elle se dira, croira « ab-ject», répétera son manque, son pas-tout ou l'agira dans le silence " équivalence de la mort », ce manque qui n'est pas le sien mais le désir de l'autre-homme : "lui " qui la veut savoir dans le manque et la veut voir dans le Tout-du sexe. "Lui » qui écrit son désir comme désir d'être .. . l'Être autoreproducteur de soi-même. Désir qui va s'écrire sur un autre événement : La mort - du corps - génératrice de l'esprit.

II. LA « MORT », LIEU D'ÉMERGENCE DE L'« ESPRIT/LOI ".

- Origine et conséquences du totémisme et de la loi de l'interdit de l'inceste. 
Si les théories précédentes se lisaient théories du corps-sexe, celle issue de la mort-événement - dite " première théorisation » - va s'écrire théorie d'elle-même : théorie de l'Espritproducteur de Toute théorie.

De sa rencontre fortuite avec la mort, l'homme va construire la première théorisation : celle des "âmes (esprits) indépendantes des corps " productrices par eux de théories/représentations d'elles-mêmes, modèle de l'auto-reproduction.

Dieu (le père-mort) avait pris la résolution de le châtrer, d'user de lui comme d'une femme et d'engendrer par lui des bommes nouveaux de l'essence des Schreber, ${ }^{12}$

telle est la pratique du principe mise ici en scène par le Président de la Haute Court de Saxe dans la forme-objet du désir de soumission du fils au père-mort/Esprit, telle est la représentation (modèle) de la nécessaire reproduction du Père/Esprit par le fils, de la nécessaire « andro-génèse ».

Le contenu de la première théorisation se dit origine et première théorie, se dit réflexivement : il dit que ce qu'il dit (fait) est identique à dire, faire, théoriser. Mais, que dit-il ?

\section{Le mythe de la Horde Primitive}

Un père violent, possédant toutes les femmes, chasse les fils afin de rester maître des femmes.

Un jour, les frères chassés se sont réunis, ont tué et dévoré le père (. . .). L'aïeul violent était certainement le modèle envié et redouté de chacun des membres de cette association fraternelle. Or, par l'acte de l'absorption, ils réalisaient leur identification avec lui, s'appropriaient chacun une partie de sa force (. . .). Après l'avoir supprimé, après avoir assouvi leur haine et réalisé leur identification avec lui (. . .) le mort devenait plus puissant qu'il ne l'avait jamais été de son vivant. Ce que le père avait empêché autrefois (. . .) les fils se le défendaient à présent euxmêmes (. . .) ils désavouaient leur acte, en interdisant la mise à mort du totem, substitut du père, et ils renonçaient à cueillir les fruits de ces actes, en refusant d'avoir des rapports sexuels avec les femmes qu'ils avaient libérées. C'est ainsi que le « sen-

12. Ibid., p. 234. L'italique est de nous. 
timent de culpabilité » du fils a engendré les deux tabous fondamentaux du totémisme qui, pour cette raison devait se confondre avec les deux désirs réprimés du "complexe d'Oedipe " (. . .). L'interdiction de l'inceste avait une grande importance pratique. Le besoin sexuel, loin d'unir les hommes, les divise. Si les frères étaient associés, tant qu'il s'agissait de supprimer le père, ils devenaient rivaux, dès qu'il s'agissait de s'emparer des femmes. Chacun aurait voulu, à l'exemple du père les avoir toutes à lui, et la lutte générale qui en serait résultée aurait amené la ruine de la société (. . .) Aussi les frères, s'ils voulaient vivre ensemble, n'avaient-ils qu'un seul parti à prendre : instituer l'interdiction de l'inceste, par laquelle ils renonçaient tous à la possession des femmes convoitées, alors que c'était principalement pour s'assurer cette possession qu'ils avaient tué le père. Ils sauvèrent ainsi l'organisation qui les avait rendus forts et qui reposait peut-être sur des sentiments et des pratiques homosexuels. ${ }^{13}$

Le contenu de la théorie originaire est le procès de production de la Loi (de l'interdit de l'inceste) ou : comment vint aux hommes l'idée de la Loi, sous quelle forme, dans quel contexte fut-elle créée, quelle est sa fonction ? En un mot, le contenu se dit cause de la Loi et, par elle, cause de la civilisation.

Reprenons la mise en scène de la Loi :

1 - Le décor est le suivant : un père violent, maître de toutes les femmes, chasse les fils-frères pour rester le Maître. Il crée, par ce geste, le dehors occupé par les fils-frères, et le dedans, opposé au premier, son lieu, lieu de la Maîtrise (des femmes) et lieu des femmes soumises au Père-Maître.

2 - L'action est le crime et la " dévoration » du père, son lieu celui de la Maîtrise et son mobile l'appropriation des femmes.

Au dehors, lieu de l'exclusion des fils-frères, ces derniers s'organisent ... en meurtriers : lieu sans femmes, ils ont (peutêtre) des sentiments et des pratiques homosexuels, cause de leur force.

Dedans, les fils-frères tuent le Père, libérant, par ce geste, les femmes, et dévorent le Père afin de s'approprier sa force . . . virile et/ou de Père. Ils «s'id-entifient " à lui.

13. Sigmund Freud, Totem et tabou, Paris, Payot 1977, p. 165. L'italique est de nous. 
Dans cette scène, par cet acte, les fils-frères sont devenus des pères et des pairs, et les femmes (toujours indifférenciées) sont libres . . . pour l'appropriation par un autre Maître, libres par et pour les fils-frères. Chacun des fils-frères devenu aussi violent que le Père est en mesure de s'approprier toutes les femmes et donc de tuer tous les autres Pères. Aussi, pour éviter la " ruine de l'organisation qui les avait rendus forts " (que les femmes restent sans Mâttre ?) ils créent la Loi - de l'interdit de l'inceste -, créent l'« exo-gamie ": les femmes sont interdites dedans mais prescrites objet d'appropriation au dehors. Ils placent ce faisant - l'objet d'appropriation au lieu qui les avait rendus forts : l'ailleurs/l'exclusion ; lieu, ne l'oublions pas, d'où viennent les meurtriers - l'autre lieu. Ils font de la volonté du Père (être le Maître des femmes) la Loi, ils donnent au Père-mort la force qu'il n'avait pas vivant : la force de Loi et, par ce don, se soumettent au Père-Loi, se soumettent à la Loi-du Père qu'ils viennent de créer. Ils font de lui leur Maître ẹt d'eux ses sujęts (attributs ?). Ils se disent - se créent - sujets du Maître-Père, semblables aux «femmes antérieures» (femmes d'avant le meurtre).

3 - Place et fonction de l'un et l'autre corps-sexué dans l'acte civilisateur. Dans cette scène, moment de la création de la Loi, les femmes deviennent semblables aux fils-frères antérieurs et ces derniers aux femmes antérieures : les femmes de la libération sont dites sans maître (dedans) et meurtrières par l'autre lieu (au dehors), et, les hommes, sujets du Père.

Si le rapport des fils-frères au Maître-Père est le même que celui des femmes antérieures, rapport de soumission, les termes en sont changés : dans un cas, des corps-femmes soumis à un Maître-vivant, dans l'autre, des corps-hommes se soumettant à un Maître-Père mort - la Loi - Dans un cas, un participe passé, dans l'autre, un participe présent qui se donne comme passé : comme " toujours déjà ».

Mais, il y a plus dans ce geste fondateur. En faisant, disant la volonté du Père la Loi - de l'interdit de l'inceste - les fils-frères re-connaissent au Père-(mort) la propriété des femmes et de la Loi. Les femmes et la Loi : les deux attributs du MaîtrePère représentés par la Loi seule . . . matrice/femme de toute 
les théorisations et/ou organisations sociales et, théorie elle-même, la Théorie.

Qu'elle soit théorie/procès est cela même qui doit s'effacer, se refouler, tout comme doivent disparaître les femmes-attribut du Père, c'est-à-dire la spécificité du rapport de l'un et l'autre corps, de l'un et l'autre sexe. Si « les femmes » s'entend comme attribut de l'autre-(corps) homme, objet d'envie (d'être) des filsfrères, c'est lire leur qualité en tant que qualité du père-autre homme (père antérieur), leur qualité antérieure. C'est lire la préhistoire du rapport de l'un et l'autre corps-sexué sous la forme de : un homme-violent (qualité interne) avec ... à lui « les femmes" (qualité externe).

Les femmes : une des deux qualités de l'homme. Telle est (écrite) par l'histoire, leur pré-histoire, leur "avoir été ", la forme-objet - construit par lui pour lui - de leur désir.

Ce contenu est à la fois posé et nié par celui constitutif de l'Histoire : meurtre et dévoration du Père par les fils-frères, cause de la Loi (de l'interdit de l'inceste) créatrice de civilisation, de théories de/sur l'autre-nature. Contenu demeuré sous le voile des femmes - en manque de maître et des fils-frères reproducteurs du Discours, du Devoir-être, de la première organisation sociale - première pratique théorique.

La Loi (de l'interdit de l'inceste), Loi-du Père, produit les fils-attribut du Maître-Père, corps-parlant la Loi, producteurs de théories, et, "les femmes ", indispensables (mais non nécessaires) à la reproduction du corps-parlant.

Les femmes sont dites à la fois : a) qualité de l'homme(père) antérieur ; b) libres - en manque de Maître, prédicat sans sujet $(!) ; c)$ objet d'appropriation des fils-frères Maître de la théorie et, en tant que telles, " chose " dangereuse (lieu de l'autre, de l'exclusion), mortifere, réceptacle, lieu de passage du corps (fils) parlant reproducteur de la Loi (producteur qui se dit reproducteur, effaçant par ce geste, ce procès, le meurtre cause de la Loi et le mobile du meurtre : l'appropriation des femmes).

Pour les fils-frères, la " noce " (-gamie) sera - toujours déjà - rencontre avec le danger, la mort ; sera toujours déjà 
appropriation du meurtrier-indispensable à la reproduction du " même ".

Pour les femmes, la " noce " sera soumission à un maître dont elles attendront d'être l'attribut : modèle de la contiguïté directe, modèle du rapport sexuel (le « rapport sexuel », comme l'écrit Lacan, à ce titre, n'existe pas) ${ }^{14}$. Elles attendront l'impossible, puisque pour l'autre-homme, l'attribut est ce qu'elles ne sont pas : "Lui » corps-(fils) parlant la Loi/théorie. Leur identité sera écrite " elles" : accumulation anarchique de toutes les représentations de l'autre-femme produites par les fils-frères théoriciens auxquelles s'ajoute le " désir » d'être . . . l'attribut, d'être fils - frères à la place des fils - nouvelle écriture sur elles, par eux, de leur désir, forme-objet (moderne) de leur désir inscrit par eux, pour elles : l'envie du pénis-(phallus) ; être à leur place, porteur de la Loi, d'être ce qu'ils disent avoir été : envieux de la place de l'autre (advenu Autre) et, de ce fait, meurtriers. Re-présentation qui fait qu'une femme ne pourra se croire - encore . . . attribut-qualité du Père qu'à n'être pas une fermme mais un fils (sous les traits d'une femme) ; représentation qui, comme la Loi dont elle est issue, condamne et prescrit à la fois : condamne l'autre-femme à n'être pas ce qui pourtant doit être . . . un fils reproducteur de la Loi du Père - représentation du Tout - mais, lieu de passage de ce dernier. Vieux rêve d'engendrement du mâle par le mâle dans un contenant approprié :

Ce n'est pas la mère dont on peut dire qu'elle enfante l'en-

fant : elle n'est que la nourrice du germe en elle semé. Celui qui enfante, c'est l'homme qui la féconde ; elle, comme étrangère, sauvegarde la jeune pousse. ${ }^{15}$

Le « drame » des fils-frères se lit dans les termes suivants : l'autre-homme condamné à $n$ 'être pas ce qui pourtant doit être . . . l'Être-absolu, la Loi-identique au Père, la Loi autogénératrice, ce que - justement - elle n'est pas puisqu'il est condamné, par elle (qu'il a créée) à être son re-producteur. Reproducteur du manque de la Loi du fait d'avoir à la re-dire, il se doit de la dire souveraine, sans rapport à lui, se doit de

14. Jacques Lacan, Séminaire $X X$, Paris, Seuil, 1975.

15. Euménides, 658, 661, cité par Nicole Loraux dans Les enfants d'Athéna, Paris, Maspéro, 1981 , p. 129. 
s'oublier. Ce que les fils-frères jouent sur la scène du Père-Loi, ils le font jouer, par les femmes, sur la leur.

Dans cette histoire - l'Histoire - où j'entends dire les femmes par les fils, il est aussi entendu que les fils-frères sont dits : ils sont dits se disant l'un-l'autre, se disant « je " disant l'autre-femme " écho " de " je ", ils sont dits se disant meurtriers, voleurs de place, impuissants à être Souverain : sans rapport (sexuel) à l'autre-femme.

Désir de l'andro-gyne sous la forme-objet d'amour de l'andro-génèse ; désir de l'impossible joué cette fois sur le théâtre de l'Esprit-(théorie) Loi - telle est la double entente du Désir (de « lui ») : désir d'être la beauté (son modèle) et désir d'avoir le pouvoir re-producteur, deux créations du rapport . . . à l'autrefemme.

Elle, attendant de lui un Maître-Père qui la reconnaisse . . . son attribut/qualité, Lui cherchant par elle à se reproduire Tout - c'est-à-dire sans elle, niant ce que justement elle attend - telle se lit/écrit la " rencontre " de l'un et l'autrecorps sexué, rencontre prescrite impossible par l'un pour l'Un ... sur l'autre-corps.

Département de philosophie

Université du Québec à Montréal 